\title{
Quantum Logic Gates and Nuclear Magnetic Resonance Pulse Sequences
}

\author{
J. A. Jones \\ OCMS, New Chemistry Laboratory, South Parks Road, Oxford, \\ OX1 3QT, UK, and Centre for Quantum Computation, Clarendon \\ Laboratory, Parks Road, Oxford OX1 3PU, UK \\ R. H. Hansen \\ Centre for Quantum Computation, Clarendon Laboratory, Parks \\ Road, Oxford OX1 3PU, UK \\ M. Mosca, Centre for Quantum Computation, Clarendon \\ Laboratory, Parks Road, Oxford OX1 3PU, UK and Mathematical \\ Institute, 24-29 St Giles', Oxford, OX1 3LB, UK \\ Correspondence should be addressed to J. A. Jones at the New \\ Chemistry Laboratory, telephone +44 1865 275923, FAX +44 1865 \\ 275921, e-mail jones@bioch.ox.ac.uk. \\ Running title: Quantum Logic Gates
}

\begin{abstract}
There has recently been considerable interest in the use of Nuclear Magnetic Resonance (NMR) as a technology for the implementation of small quantum computers. These computers operate by the laws of quantum mechanics, rather than classical mechanics, and can be used to implement new quantum algorithms. Here we demonstrate how NMR can in principle be used to implement all the elements required to build quantum computers, and briefly discuss the potential applications of insights from quantum logic to the development of novel pulse sequences with applications in more conventional NMR experiments.
\end{abstract}

Key words: NMR, quantum computer, qubit, logic gate, controlled NOT. 


\section{INTRODUCTION}

It is well known that it is difficult to simulate the behaviour of a quantum mechanical system with a classical computer. The difficulty arises because quantum systems are not confined to their eigenstates but can in general exist in any superposition of them; thus the vector space needed to describe the system is extremely large. For example a spin system comprising $N$ spin- $\frac{1}{2}$ nuclei occupies a Hilbert space with $2^{N}$ dimensions. For this reason it is impractical to simulate the behaviour of spin systems with more than about a dozen nuclei.

In 1982 Feynman (1]) reversed this observation, suggesting that quantum mechanical systems have a potentially very large information processing ability. Thus it should be possible to build quantum mechanical computers which utilise this ability to achieve a computing power well beyond that of corresponding classical systems. The theory of such quantum computers is now fairly well understood, but it has proved extremely difficult to actually build one. Recently, however, attempts to build computers based on the NMR properties of small

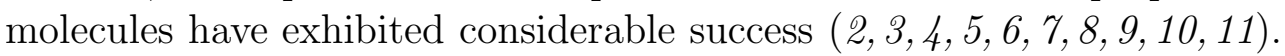

In this paper we show how NMR can be used to implement all the "components" required to construct quantum computers, and draw comparisons between quantum logic gates and more conventional NMR pulse sequences. The potential role of quantum computing as a source of new insights into NMR is also briefly addressed.

\section{$1.1 \quad$ BITS AND QUBITS}

The basic unit of information in a classical computer is the bit, which can take one of two values, 0 and 1 . Bits are then connected together by logic gates to form logic circuits, which can implement more complex logic operations, such as addition. Developments in classical computers have been driven by developments in the design and construction of logic gates, which have steadily become smaller, faster, and cheaper. However this process is beginning to reach a fundamental limit, as logic gates are reduced in size to atomic dimensions. Progress beyond this limit will require a different approach.

One obvious possibility is to implement bits and logic gates using atomic components. A bit can be implemented using any two state device, such as the two quantum states of a two level system. For example the two Zeeman levels, $|\alpha\rangle$ and $|\beta\rangle$, of a ${ }^{1} \mathrm{H}$ nucleus in a magnetic field can be naturally described as a bit. Similarly a spin-system containing $N$ distinct ${ }^{1} \mathrm{H}$ nuclei can be modelled as a set of $N$ bits. Traditionally the lower and upper energy levels are referred to as $|0\rangle$ and $|1\rangle$ respectively.

The time-evolution of a spin-system system under some Hamiltonian is described by a series of unitary transformations, and so is of necessity reversible. Hence any quantum mechanical computer can only implement reversible opera- 
tions, and must be built from reversible logic gates. This is not an important restriction as it has been shown that reversible logic gates can be used to simulate traditional gates, and thus reversible computers are just as powerful as their irreversible counterparts $(19,19)$.

There is, however, much more to quantum computers than the implementation of classical algorithms using reversible logic: quantum computers are also capable of implementing new types of quantum mechanical algorithms (14, 15, 10, 17), with potentially enormous powers. This occurs because a two-level quantum system is not confined to its two eigenstates, but can exist in superpositions of these two states; that is the system is not confined to $|0\rangle$ and $|1\rangle$, but can exist in states such as

$$
c_{0}|0\rangle+c_{1}|1\rangle
$$

where $c_{0}$ and $c_{1}$ are complex numbers and $c_{0}^{*} c_{0}+c_{1}^{*} c_{1}=1$. A nucleus in such a state is not really in state 0 or state 1 , but is in both states simultaneously. For this reason a two level quantum system is more than a simple bit, and is better described as a quantum mechanical bit, or qubit. A spin-system with $N$ nuclei contains $N$ qubits, and can be in a superposition of up to $2^{N}$ states. This ability to be in a large number of states simultaneously gives quantum computers an intrinsic parallelism, which is exploited in quantum algorithms.

\subsection{Qubits AND NMR SPIN STATES}

Traditional designs for quantum computers comprise $N$ two-level systems which are coupled to one another and have some specific interaction with the outside world (so that they can be monitored and controlled) but are otherwise isolated. NMR systems are, by contrast, rather different. In particular a typical NMR sample comprises not just one spin-system, but a very large number of copies, one from each molecule in the sample. Thus while quantum computers are usually described using Dirac's bra and ket notation, NMR systems are described using a density matrix, usually written in the product operator basis (18). While it is possible to draw close analogies between the states of traditional quantum computers and those of NMR systems, it is necessary to proceed with some caution.

\subsubsection{ONE QUBIT STATES}

A single qubit can be in either of its two eigenstates, $|0\rangle$ and $|1\rangle$, or in some linear superposition of them. Such a state is most conveniently written as a column vector in Hilbert space: for example the state described in equation 1 is written 
as

$$
|\psi\rangle=\left(\begin{array}{c}
c_{0} \\
c_{1}
\end{array}\right)
$$

The corresponding density matrix

$$
\rho=|\psi\rangle\langle\psi|=\left(\begin{array}{ll}
c_{0}^{*} c_{0} & c_{1}^{*} c_{0} \\
c_{0}^{*} c_{1} & c_{1}^{*} c_{1}
\end{array}\right)
$$

can be decomposed as a sum of the four Pauli basis states, $\frac{1}{2} E, I_{x}, I_{y}$, and $I_{z}$.

Consider first the eigenstates, $|0\rangle$ and $|1\rangle$. These correspond to the density matrices

$$
|0\rangle\langle 0|=\left(\begin{array}{ll}
1 & 0 \\
0 & 0
\end{array}\right)=\frac{1}{2} E+I_{z}
$$

and $|1\rangle\langle 1|=\frac{1}{2} E-I_{z}$ respectively. Multiples of the unit matrix can be added to density matrices at will without effecting any NMR observable in any way, and so as far as any NMR experiment is concerned the density matrix $I_{z}$ is equivalent to $|0\rangle$, while $-I_{z}$ is equivalent to $|1\rangle$. This simple approach is not, however, applicable to larger spin systems.

Next consider superpositions, such as $(|0\rangle+|1\rangle) / \sqrt{2}$, with its corresponding density matrix

$$
\left(\begin{array}{cc}
\frac{1}{2} & \frac{1}{2} \\
\frac{1}{2} & \frac{1}{2}
\end{array}\right)=\frac{1}{2} E+I_{x} .
$$

Once again multiples of the unit matrix can be ignored, and so $I_{x}$ is equivalent to $(|0\rangle+|1\rangle) / \sqrt{2}$. Similarly $|0\rangle+i|1\rangle$ is equivalent to $I_{y}$, while $|0\rangle-|1\rangle$ is equivalent to $-I_{x}$. Just as qubit eigenstates are closely related to magnetizations, superpositions are closely related to NMR coherences.

\subsubsection{TWO QUBIT STATES}

While the relationship between qubit states and NMR states is simple for one qubit (one spin systems), this relationship is much more complicated in systems with two or more qubits. Indeed the problem of creating NMR states corresponding to multi-qubit eigenstates prevented progress in the implementation of NMR quantum computers for many years.

Typically quantum algorithms start with all qubits in state $|0\rangle$, which for a 
two-qubit computer is the state $|00\rangle$. The corresponding density matrix

$$
|00\rangle\langle 00|=\left(\begin{array}{llll}
1 & 0 & 0 & 0 \\
0 & 0 & 0 & 0 \\
0 & 0 & 0 & 0 \\
0 & 0 & 0 & 0
\end{array}\right)
$$

is quite different from the thermal equilibrium density matrix

$$
I_{z}+S_{z}=\left(\begin{array}{cccc}
1 & 0 & 0 & 0 \\
0 & 0 & 0 & 0 \\
0 & 0 & 0 & 0 \\
0 & 0 & 0 & -1
\end{array}\right)
$$

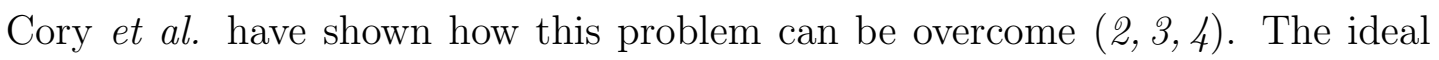
density matrix (Eq. 6) can be decomposed as the sum of four product operators:

$$
|00\rangle\langle 00|=\frac{1}{2}\left(\frac{1}{2} E+I_{z}+S_{z}+2 I_{z} S_{z}\right)
$$

and this sum (ignoring multiples of the unit matrix as usual) can be assembled using conventional NMR techniques. An alternative approach, due to Gershenfeld and Chuang (5), works by selecting four states from within a set of spin states arising from a larger spin-system. With a careful choice of states it is possible to find four levels whose relative populations correspond to those of $|00\rangle\langle 00|$, and these levels can be used as a pseudo two-spin system. While this approach is elegant, it is difficult to apply in practice and has not been widely used. A third approach, called temporal averaging ([6), is conceptually related to Cory's approach, but uses phase cycling instead of field gradients to select the desired state.

Superpositions can be treated in much the same way, but they are not directly related to coherences in any very simple way. For example consider the state $(|00\rangle+|01\rangle) / \sqrt{2}$, in which the first spin is in state $|0\rangle$, while the second spin is in a superposition of states. The corresponding density matrix can be directly decomposed:

$$
\left(\begin{array}{cccc}
\frac{1}{2} & \frac{1}{2} & 0 & 0 \\
\frac{1}{2} & \frac{1}{2} & 0 & 0 \\
0 & 0 & 0 & 0 \\
0 & 0 & 0 & 0
\end{array}\right)=\frac{1}{2}\left(\frac{1}{2} E+I_{z}+S_{x}+2 I_{z} S_{x}\right)
$$

but a more subtle approach is to note that $(|00\rangle+|01\rangle) / \sqrt{2}$ can be written as a product of one-qubit states

$$
\frac{|00\rangle+|01\rangle}{\sqrt{2}}=\frac{|0\rangle(|0\rangle+|1\rangle)}{\sqrt{2}} .
$$

The corresponding density matrix can also be decomposed as a direct product of 
equations 4 and 5 :

$$
\left(\begin{array}{ll}
1 & 0 \\
0 & 0
\end{array}\right) \otimes\left(\begin{array}{cc}
\frac{1}{2} & \frac{1}{2} \\
\frac{1}{2} & \frac{1}{2}
\end{array}\right)=\left(\frac{1}{2} E+I_{z}\right) \times\left(\frac{1}{2} E+S_{x}\right)=\frac{1}{2}\left(\frac{1}{2} E+I_{z}+S_{x}+2 I_{z} S_{x}\right) .
$$

Note that, unlike the one qubit case, a simple superposition does not correspond directly to an NMR coherence, but rather to a complex mixture of coherences and populations. Fortunately it is rarely necessary to directly consider issues of this kind, as such states can be easily obtained from states like Eq. 6.

Finally we consider superpositions of the form $(|00\rangle+|11\rangle) / \sqrt{2}$, which cannot be broken down into a product of one qubit states (such states are normally referred to as entangled states). As such states cannot be factored it is necessary to decompose the corresponding density matrices directly. In this case

$$
\left(\begin{array}{cccc}
\frac{1}{2} & 0 & 0 & \frac{1}{2} \\
0 & 0 & 0 & 0 \\
0 & 0 & 0 & 0 \\
\frac{1}{2} & 0 & 0 & \frac{1}{2}
\end{array}\right)=\frac{1}{2}\left(\frac{1}{2} E+2 I_{z} S_{z}+2 I_{x} S_{x}-2 I_{y} S_{y}\right)
$$

which is a mixture of longitudinal two-spin order and $D Q_{x}$ double quantum coherence.

\subsection{Global Phase Shifts}

One important consequence of the density matrix description of NMR quantum computers is that it is completely insensitive to global phase shifts. In general the wavefunction of any isolated system can be multiplied by an arbitrary phase shift without any observable consequences, that is the states $|\psi\rangle$ and $\left|\psi^{\prime}\right\rangle=e^{i \phi}|\psi\rangle$ are indistinguishable. Indeed the absolute value of $\phi$ is completely meaningless, although it is possible to determine relative values of $\phi$ for otherwise indistinguishable states by interference experiments.

In the density matrix description of a state such global phases are not preserved, as

$$
\left|\psi^{\prime}\right\rangle\left\langle\psi^{\prime}\left|=e^{i \phi}\right| \psi\right\rangle\left\langle\psi\left|e^{-i \phi}=\right| \psi\right\rangle\langle\psi|
$$

Thus global phases have no discernable effect in any NMR experiment, and can be completely ignored. This is fortunate, as most NMR pulse sequences create global phase shifts, as discussed below, but as such phase shifts are truly global they can be neglected.

\section{OnE QUBit GATES}

One qubit gates act to modify the spin state of a single nucleus, and thus correspond to rotations in single-spin subspaces. Any rotation of this kind can be 
achieved using RF fields, and so these gates are relatively straightforward. The gates can be implemented using selective pulses, in which case the gate is applied to a single nucleus, or using hard pulses, in which case the gate is simultaneously applied to a large number of separate nuclei. In this latter case the gate is more properly considered as a product of one qubit gates, one for each nucleus affected.

\subsection{The not GATE}

The simplest one qubit gate is the NOT gate (13), which is well known from classical computing (thus NOT is a classical one bit gate, as well as a one qubit gate). This gate, which we shall call $\mathrm{N}$, implements the rotation

$$
\begin{aligned}
& |0\rangle \stackrel{\mathrm{N}}{\longrightarrow}|1\rangle \\
& |1\rangle \longrightarrow|0\rangle
\end{aligned}
$$

This can be described more compactly using a transformation matrix

$$
\mathrm{N}-\left(\begin{array}{ll}
0 & 1 \\
1 & 0
\end{array}\right)
$$

where the symbol on the left signifies a NOT gate in a quantum circuit (19). Clearly this gate can be implemented using a $180^{\circ} I_{x}$ pulse, as

$$
e^{-i \pi I_{x}}=\left(\begin{array}{cc}
0 & -i \\
-i & 0
\end{array}\right)
$$

and this matrix has the correct form up to a global phase change. This global phase term is irrelevant, as the overall phase is not an NMR observable quantity, and thus a $180^{\circ} I_{x}$ pulse provides a good implementation of a NOT gate.

As the NOT operation is simply an inversion, it is hardly surprising that it is implemented by a $180^{\circ}$ inversion pulse. It might seem that any inversion pulse, such a $180^{\circ} I_{y}$, would be suitable, but this is not the case. For example

$$
e^{-i \pi I_{y}}=\left(\begin{array}{cc}
0 & -1 \\
1 & 0
\end{array}\right)
$$

performs the transformation

$$
\begin{aligned}
& |0\rangle \longrightarrow|1\rangle \\
& |1\rangle \longrightarrow-|0\rangle
\end{aligned}
$$

This is not a simple inversion, as it also negates the sign of the $|1\rangle$ state. This is not very important when the gate is applied to a system in an eigenstate, but is important when the gate is applied to a superposition. Consider, for example, 
the superposition $(|0\rangle+|1\rangle) / \sqrt{2}$, for which

$$
|0\rangle+|1\rangle \stackrel{\mathrm{N}}{\longrightarrow}|0\rangle+|1\rangle
$$

while a $180^{\circ} I_{y}$ pulse would give $-|0\rangle+|1\rangle$, a quite different state. This should not be surprising, as superpositions are closely related to NMR coherences, and $I_{x}$ and $I_{y}$ pulses can have quite different effects, depending on the relative phases of the pulse and the coherent state.

\subsection{ThE SQUARE-ROOT OF NOT}

The square-root of NOT gate, $\mathrm{V}$, is a purely quantum mechanical gate, in that it has no classical equivalent. As the name implies, $V$ has the property

$$
\mathrm{V}^{2}=\mathrm{VV}=\mathrm{N}
$$

and so an obvious implementation is a $90^{\circ} I_{x}$ pulse,

$$
e^{-i \pi / 2 I_{x}}=\frac{1}{\sqrt{2}}\left(\begin{array}{cc}
1 & -i \\
-i & 1
\end{array}\right) .
$$

Once again this is equal to the "ideal" form,

$$
\frac{1}{2}\left(\begin{array}{cc}
1+i & 1-i \\
1-i & 1+i
\end{array}\right)
$$

up to a global phase.

The effect of $\mathrm{V}$ is to take an eigenstate to a superposition of eigenstates. For example

$$
|0\rangle \stackrel{\vee}{\longrightarrow}(|0\rangle-i|1\rangle) / \sqrt{2}
$$

This emphasises the quantum mechanical nature of $\mathrm{V}$, as such superpositions do not have classical equivalents.

Pulses with other flip angles can be treated in much the same way: for example a $60^{\circ} I_{x}$ pulse is equivalent to a cube-root of NOT gate. This is not particularly interesting for one qubit gates, but becomes more interesting when comparing two qubit logic gates with spin state selective excitation sequences.

\subsection{The Hadamard Gate}

The square root of NOT is not unique in converting eigenstates to superpositions: any $90^{\circ}$ pulse will have a similar effect, as will a number of other pulse sequences. One particularly interesting sequence would be one corresponding to 
the Hadamard gate, $\mathrm{H}$, which performs the rotation

$$
\begin{aligned}
& |0\rangle \stackrel{\mathrm{H}}{\longrightarrow}(|0\rangle+|1\rangle) / \sqrt{2} \\
& |1\rangle \longrightarrow(|0\rangle-|1\rangle) / \sqrt{2}
\end{aligned}
$$

This has two useful properties. First, it takes $|0\rangle$ to a completely uniform superposition, that is to a state where the coefficients in front of $|0\rangle$ and $|1\rangle$ are identical. Secondly, $\mathrm{H}$ is self inverse, so applying $\mathrm{H}$ twice is equivalent to doing nothing.

$\mathrm{H}$ can be implemented in NMR using an off-resonance pulse, as

$$
e^{-i \pi\left(I_{x}+I_{z}\right) / \sqrt{2}}=\frac{1}{\sqrt{2}}\left(\begin{array}{cc}
-i & -i \\
-i & i
\end{array}\right),
$$

which has the desired form, ignoring a global phase shift as usual. Alternatively this can be implemented using a three pulse sandwich (20), such as

$$
45 I_{y}-180 I_{x}-45 I_{-y} .
$$

When implementing quantum algorithms on NMR quantum computers, it is often easier to replace the Hadamard, $\mathrm{H}$, by the pseudo-Hadamard operator, $\mathrm{h}$, which has the form

$$
h=\frac{1}{\sqrt{2}}\left(\begin{array}{cc}
1 & 1 \\
-1 & 1
\end{array}\right)
$$

This operation takes $|0\rangle$ to a uniform superposition of $|0\rangle$ and $|1\rangle$, just like $\mathrm{H}$, but it is not self inverse. This can be implemented using a $90_{y}^{\circ}$ pulse. In many algorithms, a pair of $\mathrm{H}$ gates can be replaced by one $\mathrm{h}$ gate and one $\mathrm{h}^{-1}$ gate; this last gate is easily implemented as a $90_{-y}^{\circ}$ pulse. Considered as an NMR operator, the $\mathrm{H}$ sequence performs the rotation

$$
I_{z} \stackrel{\mathrm{H}}{\longrightarrow} I_{x} \stackrel{\mathrm{H}}{\longrightarrow} I_{z}
$$

while $\mathrm{h}$ performs the more conventional rotation

$$
I_{z} \stackrel{\mathrm{h}}{\longrightarrow} I_{x} \stackrel{\mathrm{h}}{\longrightarrow}-I_{z}
$$

\section{Two QUBIT GATES}

All the gates described above are one qubit gates: in NMR terms they perform rotations within the subspace corresponding to a single spin. If implemented using hard pulses they can in general effect several spins, but the overall effect is that each spin rotates within its own subspace, and the operation can be 
decomposed as the product of two or more one spin operations. True two qubit gates correspond to rotations within a subspace corresponding to two spins, and cannot be decomposed into a set of one qubit gates. These gates lie at the heart of quantum computation, as they provide conditional dynamics: that is, the state of one spin can become dependent on the state of another spin.

\subsection{The CONTROLLED-NOT GATE}

The fundamental two qubit gate is the controlled-NOT gate, which applies a NOT gate to one qubit (the "target") if another qubit (the "control") is in state $|0\rangle$. It can be described as follows:

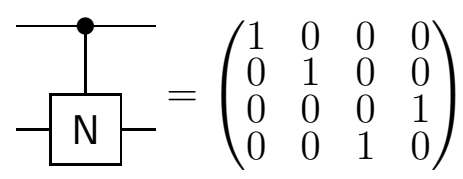

A variety of methods for implementing this gate have been described, but it is more useful to consider a general approach to gates of this kind. It is well known in quantum computation that controlled gates, such as the controlled NOT, are related to controlled phase shifts by the Hadamard transform. For example a controlled-NOT gate can be replaced by the three gate network

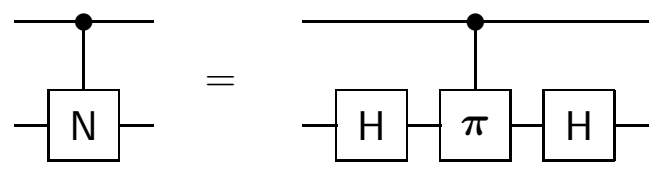

where $\mathrm{H}$ are one qubit Hadamard gates, and $\boldsymbol{\pi}$ is given by

$$
\boldsymbol{\pi}=\left(\begin{array}{cccc}
1 & 0 & 0 & 0 \\
0 & 1 & 0 & 0 \\
0 & 0 & 1 & 0 \\
0 & 0 & 0 & -1
\end{array}\right)
$$

which performs the transformation $|11\rangle \rightarrow-|11\rangle$, while leaving other basis states unchanged.

When implementing controlled gates in NMR pulse sequences, it is simpler to replace the Hadamard gates by pseudo Hadamard gates, as described above. Hence a general controlled gate can be implemented in NMR using the network

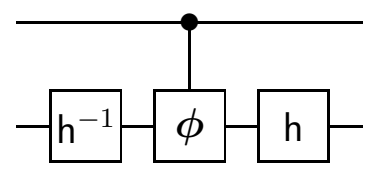


where $\phi$ is a general controlled phase shift

$$
\boldsymbol{\phi}=\left(\begin{array}{cccc}
1 & 0 & 0 & 0 \\
0 & 1 & 0 & 0 \\
0 & 0 & 1 & 0 \\
0 & 0 & 0 & e^{i \phi}
\end{array}\right)
$$

which performs $|11\rangle \rightarrow e^{i \phi}|11\rangle$, while leaving other basis states unchanged.

This general form for controlled gates in NMR should not be a surprise, as there is a close link with composite $z$-pulses. It is well known that $z$-pulses can be replaced by three pulse sandwiches (20, 21); for example a $\theta_{z}$ pulse can be replaced by $90_{-x}^{\circ} \theta_{y} 90_{x}^{\circ}$. Similarly, by cyclic permutation of axes, a $\theta_{x}$ pulse can be replaced by $90_{-y}^{\circ} \theta_{z} 90_{y}^{\circ}$, which is equivalent to $\mathrm{h}^{-1} \theta_{z} \mathrm{~h}$. Since a NOT gate (a $180_{x}^{\circ}$ pulse) can be implemented as an inverse pseudo Hadamard, followed by a $180^{\circ}$ phase shift, followed by a pseudo Hadamard, it is hardly surprising that a controlled-NOT GATE can be implemented in much the same way, but using a controlled phase shift.

\subsection{Controlled Phase Shifts}

Controlled phase shifts, such as $\phi$ (Eq. 34), are relatively simple to implement, as they can always be decomposed as a product of diagonal operators. For example

$$
\phi=\exp \left[-i \times \frac{1}{2} \phi \times\left(-\left(\frac{1}{2} E\right)+I_{z}+S_{z}-2 I_{z} S_{z}\right)\right] .
$$

The last three terms are straightforward, but the first term is difficult to obtain as it requires a Hamiltonian proportional to $\frac{1}{2} E$. This is not, however, important, as this term simply imposes a global phase shift, and as such can be ignored. For the remaining three terms, $2 I_{z} S_{z}$ is proportional to the scalar coupling Hamiltonian, while $I_{z}$ and $S_{z}$ can be implemented as periods of free precession or by using composite $z$-pulses.

The matrix $\boldsymbol{\pi}$, which lies at the heart of the controlled-NOT gate, can be implemented as

$$
\left(90^{\circ} I_{z}\right)\left(90^{\circ} S_{z}\right)\left(-90^{\circ} 2 I_{z} S_{z}\right)
$$

which can itself be achieved in a variety of ways. The three terms above commute, and so the three Hamiltonians can be applied in any order, while the $I_{z}$ and $S_{z}$ terms can be implemented by free precession or by any of a wide variety of different composite pulses.

Just like simple one qubit gates, two qubit controlled gates can also introduce global phase shifts, but as long as these are global and universal, that is they

are applied to the the whole wavefunction and not just to the spins participating in the gate and they are applied irrespective of the state of the control bit, such phase shifts can be ignored. This is indeed the case: conceptually these phase 
shifts can be thought of as arising from the lack of a $\frac{1}{2} E$ term in controlled phase shift gates, and thus have the desired properties.

\subsection{The CONTROLled SqUARE-ROOT OF NOT}

Implementation of the controlled square-root of NOT gate is simple using the approach outlined in equations 33 and 34, with $\phi=\pi / 2$. The controlled phase shift is simply implemented as

$$
\left(45^{\circ} I_{z}\right)\left(45^{\circ} S_{z}\right)\left(-45^{\circ} 2 I_{z} S_{z}\right)
$$

Note that there is a close relationship between this gate and the spin-state selective excitation sequences (22, 23) which have been suggested as a method for simplifying E-COSY spectra. Clearly any other spin-state selective pulse can be created in much the same way.

\section{Three QUBIT GATES}

A wide variety of three bit gates have been investigated, but we will confine our discussions to the Toffoli gate (13), or controlled-controlled-nOT. This takes the form

$$
\text { P }=\left(\begin{array}{llllllll}
1 & 0 & 0 & 0 & 0 & 0 & 0 & 0 \\
0 & 1 & 0 & 0 & 0 & 0 & 0 & 0 \\
0 & 0 & 1 & 0 & 0 & 0 & 0 & 0 \\
0 & 0 & 0 & 1 & 0 & 0 & 0 & 0 \\
0 & 0 & 0 & 0 & 1 & 0 & 0 & 0 \\
0 & 0 & 0 & 0 & 0 & 1 & 0 & 0 \\
0 & 0 & 0 & 0 & 0 & 0 & 0 & 1 \\
0 & 0 & 0 & 0 & 0 & 0 & 1 & 0
\end{array}\right)
$$

and plays a central role in the theory of classical reversible computation as it can be shown to be universal (that is, any reversible classical logic circuit can be constructed entirely out of classical Toffoli gates).

It might seem that this gate could be implemented using double-controlled phase shifts,

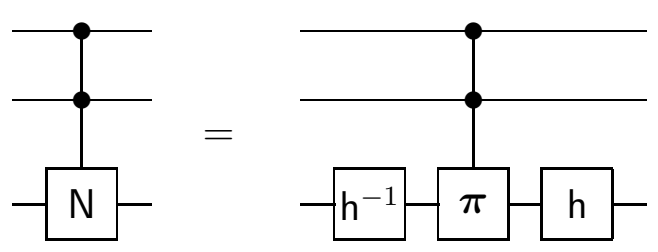

but while the above circuit is indeed correct this approach is not practical. The 
double-controlled phase shift matrix, $\boldsymbol{\pi}$ can be decomposed as

$$
\boldsymbol{\pi}=\exp \left[-\frac{i \pi}{2}\left(\frac{1}{2} E-I_{z}-R_{z}-S_{z}+2 I_{z} R_{z}+2 I_{z} S_{z}+2 R_{z} S_{z}-4 I_{z} R_{z} S_{z}\right)\right]
$$

but this decomposition cannot be used as a guide to implementing $\boldsymbol{\pi}$, as there is no NMR Hamiltonian directly corresponding to $4 I_{z} R_{z} S_{z}$. For the same reason it is not possible to directly implement a double-controlled square-root of NOT, that is a doubly spin state selective excitation sequence.

It is, however, possible to implement these gates by using more complex networks of logic gates. Indeed it has been shown that the combination of a controlled-NOT gate and a set of general one bit gates is universal (24), so that any other gate can be constructed from them. This process is even simpler if the set of basic gates is slightly expanded; for example a Toffoli gate can be implemented using the network

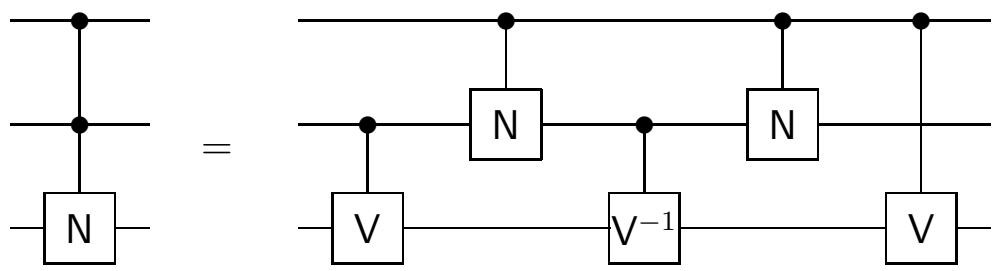

It should be noted that while it will be difficult to construct a true Toffoli gate it is relatively simple to construct an approximate Toffoli gate, whose transformation matrix has the same underlying form as equation 38, but where the non-zero matrix elements are not all equal to unity. Such a gate was demonstrated early on (因), and can be used instead of a true gate in some situations where it is the last gate in the logic network, such as error correction (29).

\section{Applying LOGiC NETWORKS IN NMR}

Having shown that NMR pulse sequences can be used to implement all the basic logic gates needed both for classical reversible computation and for quantum computation, it is instructive to consider whether any more complex logic networks might correspond to interesting NMR pulse sequences. One obvious candidate is the double-controlled square-root of NOT circuit, which corresponds to a doubly spin-state selective excitation sequence. More generally this family of gates involves the creation of an effective Hamiltonian containing a term proportional to $4 I_{z} R_{z} S_{z}$, which is not easily accessible by conventional means, and may be useful in the generation of multiple quantum coherences. 
Another logic network which might prove useful is the SWAP network

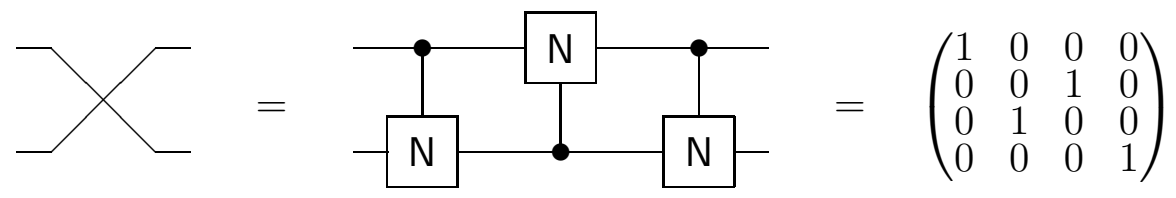

which completely interchanges the states of the two spins involved. Clearly this is closely related to sequences like the double INEPT transfer step used in sensitivity enhanced HSQC experiments (20, 27, 28). Unlike conventional heteronuclear transfer steps, however, this sequence preserves the states of both spins, by performing a complete swap.

More speculatively it may be possible to use quantum error correction codes (29, 30, 31) to reduce the effects of spin-spin relaxation upon NMR spectra. Initial experiments in this direction (25) suggest that error correction does work in NMR experiments, but that it is unlikely to have much practical significance; this assessment may, however, prove too pessimistic.

\section{ACKNOWLEDGMENTS}

This is a contribution from the Oxford Centre for Molecular Sciences which is supported by the UK EPSRC, BBSRC and MRC. JAJ thanks C. M. Dobson (OCMS) for his encouragement. RHH thanks the Danish Research Academy for financial assistance. MM thanks CESG (UK) for their support. 


\section{REFERENCES}

1. R. P. Feynman, Int. J. Theor. Phys. 21, 467 (1982).

2. D. G. Cory, A. F. Fahmy, and T. F. Havel, in T. Toffoli , M. Biafore, and J. Leão, PhysComp '96, New England Complex Systems Institute (1996) $87-91$.

3. D. G. Cory, A. F. Fahmy, and T. F. Havel, Proc. Natl. Acad. Sci. USA 94, 1634 (1997).

4. D. G. Cory, M. D. Price, and T. F. Havel, Physica D, in press. LANL e-print available from http://xxx.lanl.gov/abs/quant-ph/9709001.

5. N. A. Gershenfeld and I. L. Chuang, Science 275, 350 (1997).

6. E. Knill, I. Chuang, and R. Laflamme. LANL e-print available from http://xxx.lanl.gov/abs/quant-ph/9706053.

7. J. A. Jones and M. Mosca, J. Chem. Phys., in press. LANL e-print available from http://xxx.lanl.gov/abs/quant-ph/9801027.

8. I, L. Chuang, L. M. K. Vandersypen, X. Zhou, D. W. Leung, and S. Lloyd, Nature, 393, 143-146 (1998).

9. I. L. Chuang, N. Gershenfeld, and M. Kubinec, Phys. Rev. Lett., 80, 3408-3411 (1998).

10. J. A. Jones, M. Mosca, and R. H. Hansen, Nature, in press.

11. J. A. Jones, Science, 280, 229 (1998).

12. C. H. Bennett, IBM J. Res. Develop. 17, 525 (1973).

13. R. P. Feynman, "Feynman Lectures on Computation" (A. J. G. Hey and R. W. Allen, Eds.), Addison-Wesley, Reading, MA (1996).

14. D. Deutsch and R. Jozsa, Proc. R. Soc. Lond. A 439, 553 (1992).

15. A. Ekert and R. Jozsa, Rev. Mod. Phys. 68, 733 (1996).

16. L. K. Grover, Phys. Rev. Lett. 79, 325 (1997).

17. R. Cleve, A. Ekert, C. Macchiavello, and M. Mosca, Proc. R. Soc. Lond. A 454, 339 (1998).

18. O. W. Sørensen, G. W. Eich, M. H. Levitt, G. Bodenhausen, and R. R. Ernst, Prog. NMR Spectrosc. 16, 163 (1983). 
19. D. Deutsch, Proc. R. Soc. Lond. A 425, 73 (1989).

20. R. R. Ernst, G. Bodenhausen, and A. Wokaun, "Principles of Nuclear Magnetic Resonance in One and Two Dimensions," Clarendon Press, Oxford (1987).

21. R. Freeman, T. A. Frenkiel, and M. H. Levitt, J. Magn. Reson. 44, 409 (1981).

22. A. Meissner, J. O. Duus, and O. W. Sørensen, J. Biomol. NMR 10, 89 (1997).

23. A. Meissner, J. O. Duus, and O. W. Sørensen, J. Magn. Reson. 128, 92 (1997).

24. A. Barenco. C. H. Bennett, R. Cleve, D. P. DiVincenzo, N. Margolus, P. Shor, T. Sleator, J. A. Smolin, and H. Weinfurter, Phys. Rev. A 52, 3457 (1995).

25. D. G. Cory, W. Mass, M. Price, E. Knill, R. Laflamme, W. H. Zurek, T. F. Havel, and S. S. Somaroo, submitted to Phys. Rev. Lett. LANL e-print available from http://xxx.lanl.gov/abs/quant-ph/9802018.

26. J. Cavanagh and M. Rance, J. Magn. Reson. 88, 72 (1990).

27. A. G. Palmer III, J. Cavanagh, P. E. Wright, and M. Rance, J. Magn. Reson. 93, 151 (1991).

28. L. E. Kay, P. Keiffer, and T. Saarinen, J. Am. Chem. Soc. 114, 10663 (1992).

29. P. W. Shor, Phys. Rev. A 52, R2493 (1995).

30. A. Steane, Proc. R. Soc. Lond. A 452, 2551 (1996).

31. A. Steane, Phys. Rev. Lett. 78, 2252 (1997). 